Revista de MATEMÁticA: TeORÍA y APliCACIONEs 2016 23(1) : 241-253

CIMPA - UCR ISSN: 1409-2433 (PRINT), 2215-3373 (ONLINE)

\title{
PROYECCIONES DEMOGRÁFICAS Y ACTUARIALES POR MEDIO DEL MÉTODO DE CADENAS DE MARKOV CON MONTE CARLO
}

DEMOGRAPHIC AND ACTUARIAL PROJECTIONS
BY MEANS OF MONTE CARLO MARKOV CHAINS

Ismael Morales GaraY* MARlyn CAStro Esquivel $^{\dagger}$

Received: 28 Feb 2012; Revised: 26 Jun 2015;

Accepted: 30 Sep 2015

\footnotetext{
*Escuela de Matemática, Universidad de Costa Rica \& Junta de Pensiones y Jubilaciones del Magisterio Nacional, San José, Costa Rica. E-Mail: imorales@juntadepensiones.cr

${ }^{\dagger}$ Misma dirección que/Same address as:. E-Mail: mcastro@juntadepensiones.cr
} 


\title{
Resumen
}

Se presenta una descripción del uso del método de las Cadenas de Markov por Monte Carlo para realizar las proyecciones demográficas actuariales, tomando como insumo las tablas de mortalidad, invalidez, mortalidad para inválidos para la población activa del fondo de pensiones del Magisterio Nacional de Costa Rica. Se incluyen ejemplos de la forma en que se implementa el método en la Junta de Pensiones y Jubilaciones del Magisterio Nacional de Costa Rica, que son parte de la información clave para la realización de las Valuaciones Actuariales del Régimen de Pensiones de los educadores.

Palabras clave: simulación Monte Carlo; cadenas de Markov; probabilidades; demografía; proyecciones actuariales.

\begin{abstract}
A description of the method of Monte Carlo Markov Chains is presented for actuarial demographic forecast, in regard to the mortality, disability, mortality of disabled lives among others. We include examples of how the method is implemented in the Pensions and Retirements Fund for Teachers in Costa Rica, as a input for the results of the actuarial valuation of the pension plan.
\end{abstract}

Keywords: Monte Carlo simulation; probability; demography; actuarial forecast.

Mathematics Subject Classification: 60J22, 65C05.

\section{Introducción}

La Junta de Pensiones y Jubilaciones del Magisterio Nacional se crea desde el año 1958, con la promulgación de la ley 2248 [1]. Su principal función es la de administrar el Régimen de Pensiones de los trabajadores de la educación, y para ello desde el año 2000 constituyó un Departamento Actuarial, el cual funge como ente técnico en la toma de decisiones. En el Magisterio Nacional existen dos regímenes de pensiones, el Régimen Transitorio de Reparto (RTR), que es un régimen cerrado y financiado por el presupuesto nacional de la república; y el Régimen de Capitalización Colectiva (RCC), el cual es administrado en su totalidad por la Junta de Pensiones y Jubilaciones del Magisterio Nacional (JUPEMA). A diciembre del 2011, el Régimen de Capitalización Colectiva contaba con 492 pensionados, y un aproximado de 70 mil afiliados activos. En este artículo cuando se cite el "Fondo", se referirá al Régimen de Capitalización Colectiva. 
En general el Fondo brinda beneficios por vejez, invalidez y sucesión o a los sobrevivientes tales como viudas(os) u orfandad, los beneficios por vejez se adquieren al obtener ciertos requisitos previos que contemplan edad mínima y número de cuotas, se le llaman también derechos por jubilación pues el afiliado obtiene el beneficio una vez que ha cumplido con su "carrera" docente o administrativa en el Magisterio Nacional. Los beneficios por invalidez y sucesión son contingencias que están sujetas a las tablas de probabilidad y por las cuales se brindan beneficios llamados pensiones, tal beneficio se estima con base al monto de una jubilación, es decir, cada Régimen de Pensiones cuenta con una metodología para calcular el monto de la jubilación, para el caso del Fondo, se obtiene el promedio de la totalidad de los salarios devengados actualizados con el Índice de Precios al Consumidor y se otorga un porcentaje de este promedio el cual se denomina cuantía base. Adicionalmente este monto se puede incrementar por cada cuota adicional superadas las 240 cotizaciones en un $0.1 \%$, lo cual se define como un porcentaje de bonificación. Si el trabajador continua laborando una vez cumplidos los requisitos edad y cuotas, puede incrementar el monto de la pensión por haber postergado su retiro en una cuantía de $1.5 \%$ por cada trimestre adicional de cotización hasta un máximo de 4 años. Asimismo, una pensión por sobrevivencia calcula con base en el monto de pensión por invalidez que hubiera devengado al momento de la muerte el causante, y no posee pluses como el de la postergación, aunque sí el de bonificación.

Para realizar la Evaluación Actuarial del Fondo, se ha creado un sistema llamado SIVA (Sistema de Valuaciones Actuariales), el cual utiliza como insumo las tablas de probabilidad de mortalidad, invalidez, mortalidad siendo inválido, escolaridad de beneficiarios, nupcias, densidad de cotización, salida del régimen, postergación de tiempo, dependencia, natalidad, reingreso entre otras, es decir, las probabilidades de acuerdo a la edad y el sexo que tiene la población para cada caso mencionado. El SIVA utiliza el método de Cadenas de Markov basados en el modelo de Monte Carlo para simular o asignar los decrementos de cada uno de estos grupos mencionados anteriormente. En este artículo se explicará la metodología que utiliza el SIVA para generar estas posibles contingencias o salidas que impactarán en la población inicial y futura a valuar.

\section{Teoría de decrementos múltiples}

Todos los derechos, tanto los de jubilación, como los de pensión, están sujetos a que los afiliados cumplan con ciertos requisitos indicados en las tablas de retiro dadas en el reglamento del fondo y en cada caso estarán sujetas a unas tablas de probabilidad, es decir del total de la población activa del régimen se irán 
generando grupos de pensionados por vejez, invalidez o sucesión. Si observamos un grupo de activos a una fecha corte, y partimos del supuesto de que no se pueden incorporar más afiliados al régimen, entonces se habla de una población de riesgo cerrada, caso contrario donde hay entrada de nuevos cotizantes, se le llama grupo de riesgo abierto. Cualquiera de estos grupos se irá disminuyendo de acuerdo con las causas de salida ya sea jubilación, invalidez o muerte. En la teoría actuarial, cuando una persona o individuo sale de un grupo por alguna causa, ya sea invalidez, jubilación u otra, se le llama decremento. (Véase [4] y [3]. El ejemplo clásico de salida de un grupo de riesgo, es aquel individuo que teniendo una contingencia resulta incapacitado para trabajar (o sea se invalida), entonces el Fondo le brinda una prestación, que puede ser monetaria o en especie, para que cubra sus necesidades básicas. Para estimar cuantas personas salen del grupo original por cierta causa, se debe suponer una probabilidad de ocurrencia del evento que depende en general de la edad y el sexo. Se debe mencionar que la ecuación básica de equilibrio actuarial del fondo de pensiones del magisterio se basa en un modelo de edad entera (véase [2]) y el modelo de financiamiento del Régimen de Capitalización Colectiva, es el explicado en [7] llamado de capitalización pura.

\subsection{Probabilidades de decremento}

En el capítulo 10 de [3], se indican básicamente cuatro fuerzas de decremento, a saber: separación del servicio, muerte en servicio, retiro por invalidez y retiro por jubilación. Las notaciones para estas probabilidades de decremento para un individuo de edad $x$ dentro de un lapso de $k$ años son ${ }_{k} q_{x}^{(w)},{ }_{k} q_{x}^{(d)},{ }_{k} q_{x}^{(i)}$ y ${ }_{k} q_{x}^{(r)}$, en donde $w$ indica renuncia o separación del servicio, $d$ es muerte en servicio, $i$ es retiro por invalidez y $r$ retiro por jubilación. Si utilizamos la notación de $l_{x}^{(\tau)}$ la función de supervivencia o estadía dentro del grupo entonces tenemos que:

$$
l_{x+1}^{(\tau)}=l_{x}^{(\tau)}\left[1-\left(q_{x}^{(w)}+q_{x}^{(d)}+q_{x}^{(i)}+q_{x}^{(r)}\right)\right]=l_{x}^{(\tau)} p_{x}^{(\tau)}
$$

donde $p_{x}^{(\tau)}$ es la probabilidad de sobrevivencia de un individuo de edad $x$ durante 1 año, esto es que no fallece, ni se invalida, ni se retira ni renuncia al servicio durante ese año. Además de estas probabilidades de decremento múltiples, podemos hacer la diferencia entre aquellas probabilidades de decremento netas, o sea que están sujetas a una causa particular únicamente, así si un afiliado de edad $x$ tiene que abandonar el grupo en los próximos $k$ años a consecuencia de una causa $j$, estando sujeto únicamente a esa causa, lo denotamos como ${ }_{k} q_{x}^{\prime(j)}$, para $j=w, d, i, r$ en particular. 


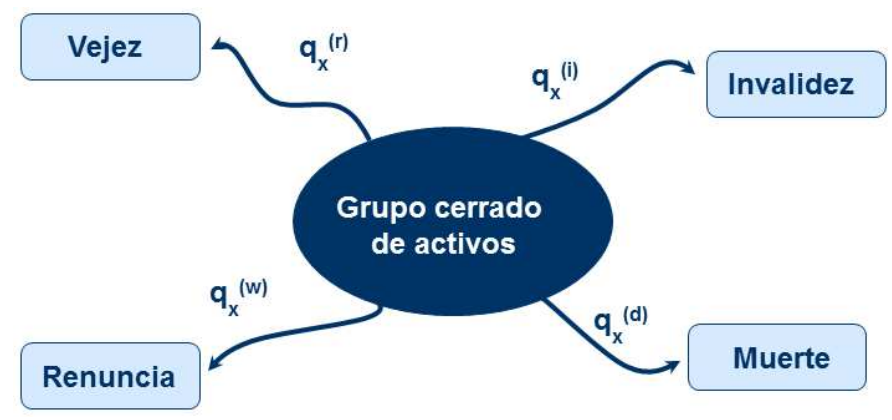

Figura 1: Fuerzas de decremento.

Cabe mencionar que dentro del fondo existen otras causas para que un afiliado activo renuncie al régimen, (por ejemplo traslado de régimen), que regrese al régimen (debido a que el nombramiento había cesado y tiempo después lo vuelven a nombrar o que un pensionado inválido se rehabilite y se reincorpore a laborar), sin embargo para efectos de este artículo, solo se utilizarán tres causas de salida: vejez, invalidez y muerte.

\subsection{Algunas relaciones básicas}

En el Sistema de Valuaciones Actuariales, se presenta el modelo de decrementos netos, toda vez que se evalúa cada riesgo por separado. Así en particular tenemos algunas relaciones que se utilizan en el sistema:

$$
{ }_{k} p_{x}^{(\tau)}=\prod_{j=1}^{n}{ }_{k} p_{x}^{(j)}=\prod_{j=1}^{n}\left(1-{ }_{k} q_{x}^{(j)}\right) .
$$

Pero como vamos a tratar solo tres causas de salida en este artículo, reescribimos la ecuación anterior como

$$
{ }_{k} p_{x}^{(\tau)}={ }_{k} p_{x}^{(d)} \cdot{ }_{k} p_{x}^{(i)} \cdot{ }_{k} p_{x}^{((r)}=\left(1-{ }_{k} q_{x}^{\prime(d)}\right)\left(1-{ }_{k} q_{x}^{(i)}\right)\left(1-{ }_{k} q_{x}^{\prime(r)}\right)
$$

Para realizar las Valuaciones Actuariales, es importante conocer la definición de la renta de vida vencida como a aquel pago periódico que se hace a una persona mientras esté con vida, el pago se llama vencido porque se paga al inicio del periodo respectivo,

$$
\ddot{a}_{x}=\sum_{k=0}^{w} v_{k}^{k} p_{x}
$$


donde $v^{k}=\frac{1}{(1+i)^{k}}$ es el valor presente de una unidad monetaria pagable $k$ periodos después (véase [5]; $\mathrm{y}_{k} p_{x}$ es la probabilidad que el beneficiario de edad $x$ permanezca vivo $k$ periodos e $i$ es la tasa de descuento.

Para efectos de este artículo, todas las rentas son vitalicias, así para un derecho por invalidez, la renta se paga mientras el beneficiario siga con vida; igual argumento si corresponde a una jubilación (pensión por vejez) se pagara durante toda la vida del beneficiario. Aunque estas rentas se pueden heredar a otros beneficiarios posterior a la muerte de un pensionado o jubilado, para efectos de nuestro artículo obviaremos este detalle técnico.

\section{Beneficios del fondo}

En [8] se indica que hay dos tipos de planes de pensiones básicos: de contribución definida y de beneficio definido. Un plan de pensiones de contribución definida, como su nombre lo indica, tiene montos definidos de cotización para el empleador y el empleado, que por lo general se calculan como un porcentaje sobre el salario nominal ganado por el empleado; el plan de beneficio definido es aquel en donde la fórmula de cálculo del beneficio o pensión está previamente definido y generalmente es una proporción del salario y años de servicio del trabajador. El Régimen de Capitalización Colectiva es un fondo de beneficio definido, pues la fórmula de cálculo de la pensión está definida en el reglamento del Régimen.

Básicamente para el cálculo de la prestación, en este Fondo, se calcula lo que se llama salario de referencia, el cual es el promedio simple de todos los salarios del trabajador actualizados por inflación. El salario de referencia es el monto base para el cálculo, el cual se multiplica por una tasa de reemplazo para obtener el monto del beneficio.

Si suponemos que el salario anual de un afiliado de edad $x+h$ es $(A S)_{x+h}$ y $(E S)_{x+h+t}$ es el salario proyectado a la edad de $x+h+t$, y si suponemos que la escala salarial 11 utilizada para la proyección es $S_{y}$ obtenemos:

$$
(E S)_{x+h+t}=(A S)_{x+h} \frac{S_{x+h+t}}{S_{x+h}} .
$$

Nótese que en el caso del Fondo esta tarea de proyección salarial se puede hacer en periodos distintos al año, tomando la escala salarial anual y dividiendo respectivamente por el factor de conversión al periodo requerido. Para valuar

\footnotetext{
${ }^{1}$ En el factor que se utiliza para actualizar el salario de una cohorte de trabajadores y que depende de la antigüedad del afiliado.
} 


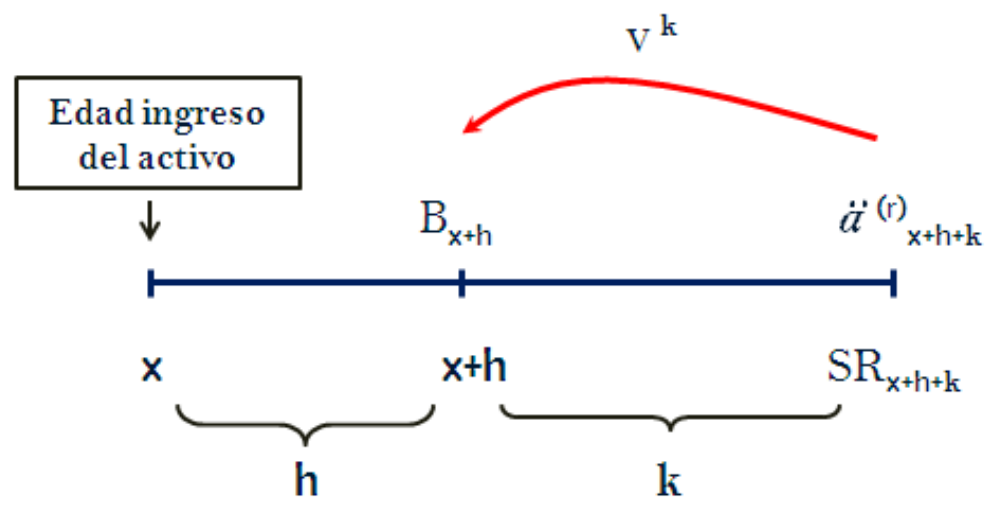

Figura 2: Valor presente actuarial del beneficio futuro.

el fondo, se deben establecer fórmulas de cálculo de los beneficios futuros con el propósito de estimar los costos esperados y así comparar con los ingresos esperados. En este sentido, para calcular el beneficio futuro de un afiliado se debe estimar el momento en que éste tiene los requisitos para jubilarse, o se invalida, o se retira del fondo. En términos simples el valor presente actuarial del costo de un beneficio $B_{t}$ tendría la forma

$$
B_{x+h}=\left[v^{k}\right]\left[t_{r}\right]\left[S R_{x+h+k}\right]\left[\ddot{a}_{x+h+k}^{(r)}\right]
$$

donde $t_{r}$ representa la tasa de reemplazo, $S R_{x+h+k}$ el salario de referencia en el tiempo $x+h+k, \ddot{a}_{x+h+k}^{(r)}$ es una renta asociada a una pensión por vejez de un activo que entró a laborar a la edad $x$ y $v^{k}$ el índice de actualización. En la Figura 2 se representa la gráfica de este beneficio.

Para el cálculo de la renta como en (4) se usa el término ${ }_{k} p_{x}$, el cual está determinado por las probabilidades de muerte, en este sentido es que el SIVA utiliza el método de Monte Carlo para distribuir esas muertes y de hecho para hacer muchos otros cálculos que se refieren a las proyecciones demográficas de cada uno de los participantes del fondo.

\section{Método de Monte Carlo}

Cuando nos referimos al método Cadenas de Markov por Monte Carlo, hacemos una relación directa con los números aleatorios o pseudoaleatorios independientes e idénticamente distribuidos. Esta forma de modelar se basa en la generación de números aleatorios que serán comparados con la probabilidad de ocurrencia de alguna contingencia o fenómeno natural y decidir si el evento se dará o no en ese momento, y así se simulará la distribución de éste evento. 
En el pasado los números aleatorios se generaban de forma manual o mecánica utilizando métodos muy rudimentarios, hoy en día, con la ayuda de la tecnología, la generación de éstos números se logra realizar de manera algorítmica en el computador de forma eficaz y velozmente. Por ejemplo, como se indica en [6], el número aleatorio se puede generar por medio del método congruencial multiplicativo, el cual inicia con una semilla $x_{0}$ y luego de forma recursiva, se obtienen los valores sucesivos $x_{n}$. Se sigue el siguiente procedimiento:

$$
x_{n}=a x_{n-1} \quad \bmod (m)
$$

en donde $a$ y $m$ son enteros positivos dados y $a x_{n-1}$ se divide entre $m$ y el residuo que genere tal división es $x_{n}$.

No obstante, en el estudio se utiliza el aleatorio que contiene el programa de Excel. Para este caso en particular se desea generar el valor de variables aleatorias discretas $X$ que siguen una distribución de tipo Bernoulli, es decir, solamente tienen dos posibles resultados:

- 1 el éxito;

- 0 el fracaso.

Es decir, se trata de simular si una persona está o no viva, está o no casada, está o no invalida, tiene o no tiene hijos, entre otros eventos que se mencionaron anteriormente. Este tipo de modelo se llama binario y conlleva a la generación de una cadena de markov, en donde cada nodo es independiente del anterior.

Una debilidad del procedimiento tradicional de proyecciones demográficas, es que cuando son poblaciones pequeñas al multiplicar la población por su probabilidad del riesgo, puede suceder que el número resultante sea difícil de interpretar por no ser un número entero. Este problema se evita cuando utilizamos el método de la cadenas de Markov por Monte Carlo para simular la población proyectada, ya que siempre nos da un número entero que representa quienes sobreviven al riesgo o por ende lo que no. Por ello si $X$ tiene la función de densidad:

$$
\operatorname{Pr}(X=0)=q_{x} ; \operatorname{Pr}(X=1)=p_{x}=\left(1-q_{x}\right),
$$

denotandos:

- $q_{x}$ la probabilidad de que una persona sufra un evento en el siguiente año, ya sea la muerte, invalidez, entre otras.

- $p_{x}$ el complemento de la probabilidad de tal evento, es decir, que no lo sufra. 
Cuando generamos el número aleatorio $\mathrm{U}$, el cual está distribuido uniformemente en $(0,1)$ para generar la simulación se plantea de la siguiente forma:

$$
X= \begin{cases}0 & \text { si } U \leq q_{x} \\ 1 & \text { si } U>q_{x}\end{cases}
$$

Esto se realiza con toda la población para una edad y género respectivo. Se obtiene la probabilidad de morir al año siguiente y para cada persona se genera un número aleatorio, por tanto se contabilizan el número de veces que el número aleatorio dio menor a la probabilidad de morir y ésta cantidad representa las personas muertas para el final del año respectivo. El mismo procedimiento se hace para toda la población en estudio con todas las probabilidades de contingencia. El proceso se realiza un número de determinado de veces (Monte Carlo), para así tomar como escenario esperado el promedio de todos los ensayos. La función de asignación se corre cientos de veces y se escoge el evento que más se dió, para así decidir si el afiliado cae en cierta contingencia.

\section{Proyecciones demográficas}

En esta sección se expondrá una aplicación del método de Cadenas de Markov por modelo Monte Carlo -revisado en el apartado anterior- para realizar proyecciones demográficas basadas en tablas de mortalidad, invalidez o en general de cualquier otro decremento. Como se indicó anteriormente el método de Monte Carlo se utiliza bajo la premisa de que estamos bajo una distribución de Bernoulli. Así dentro del SIVA, si tenemos una tabla de mortalidad que establece las probabilidades de muerte para cada edad específica, se procede a generar un número aleatorio en $(0,1)$, el cual es comparado con la probabilidad de morir ese año para un individuo de cierta edad.

En la figura 3, se presenta una aplicación del método de Cadenas de Markov sobre una probabilidad de muerte, que decide si el individuo se muere o sobrevive a ese riesgo. Así de acuerdo a la gráfica presentada en la figura 1, si generamos un número aleatorio, el cual hemos llamado $v$, vemos que $v \in\left[0, q_{x}\right]$, lo cual implica, que para ese evento, el individuo sucumbe ante ese riesgo de muerte o sea se decide que muera en esa iteración. Por el contrario, por ejemplo vemos que el otro número aleatorio $u$ cumple que $v \in\left(q_{x}, 1\right]$ lo cual nos indicaría que el individuo sobrevive a este riesgo. Nótese que esta comparación se hace para cada individuo del grupo de riesgo, para cada periodo y para cada edad correspondiente.

De acuerdo con la técnica actuarial, se realizaron varias simulaciones que demostraron la efectividad del método de cadenas de Markov por Monte Carlo 


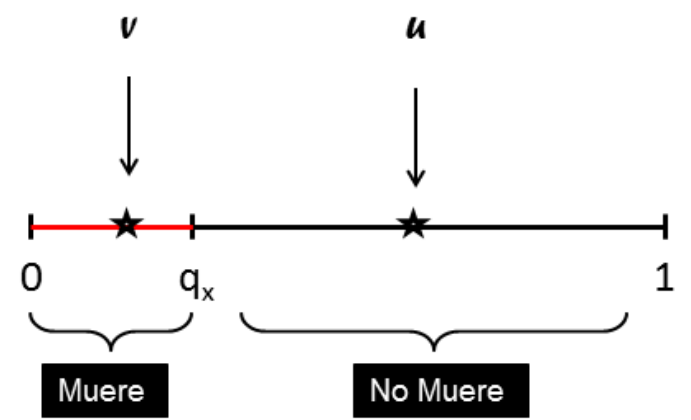

Figura 3: Aplicación del método de cadenas de Markov.

en la generación de proyecciones demográficas. Presentamos algunas muestras de lo acontecido en las pruebas.

En la figura 4, se representan gráficamente los resultados de un experimento con 1,000 valores generados por el método Monte Carlo, en donde se compara el valor esperado de $100,000 \cdot 10 p_{20}$, que indica el número de sobrevivientes de una cohorte de 100,000 individuos de edad 20, después de asumir el riesgo de muerte por 10 años contra los 1,000 valores simulados.

Los resultados del experimento se resumen en la Tabla 1 y básicamente se puede observar que el valor promedio de todos los valores Monte Carlo es exactamente igual al valor esperado, pues $100,000 \cdot{ }_{10} p_{20}=98,748.39$ y el promedio es $98,748.13$.

\begin{tabular}{c|ccc}
\hline \hline & & Diferencia & Error Relativo \\
\hline Valor Máximo & 99,065 & 317 & $0.32 \%$ \\
Valor Mínimo & 98,346 & -402 & $-0.41 \%$ \\
Promedio & $98,748.13$ & 0 & $0.00 \%$ \\
Valor Esperado & $98,748.39$ & & \\
\hline \hline
\end{tabular}

Tabla 1: Comparación de valores Monte Carlo contra valor esperado. 


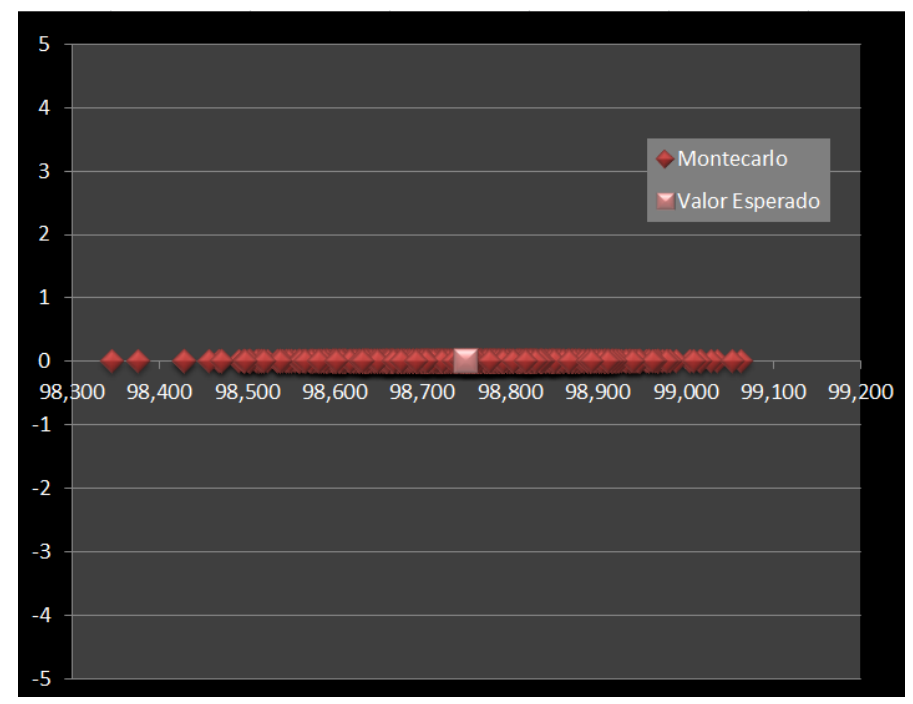

Figura 4: Simulación Monte Carlo vrs valor esperado.

Las proyecciones demográficas sobre poblaciones de riesgo cerrado que se hacen en la Valuación del Fondo se hacen al menos a 100 años plazo, en algunas pruebas preliminares que se han hecho previo a la elaboración del SIVA, se pudo constatar que los valores Monte Carlo comparados con los valores esperados eran sumamente cercanos. Sin embargo, con el método Monte Carlo hemos podido generar no solo proyecciones demográficas, sino se han generado: nuevos futuros activos del plan de pensiones, dependientes de los pensionados y activos cuando ha sido necesario o por falta de información de los mismos, simulación de postergación y retiro, entre otras.

En la Tabla2 2 se presenta una proyección elaborada para la Evaluación Actuarial del 2011, la cual se trabajó totalmente en el SIVA. Para efectos de simplicidad se presentan solo algunos rubros destacados de la proyección. El sistema realiza todos los cálculos necesarios para decrementar la población inicial de activos y además proyecta la vida de los pensionados hasta su extinción total. Asimismo, observemos que los pensionados los hemos segregado por tipo de derecho (vejez, invalidez, sucesión) y también por tipo de generación (Inicial, Actual y Futura).

Quizá el modelo tenga el inconveniente de que no es tan rápido, porque para cada individuo se debe generar un número aleatorio y así hacer la comparación con la probabilidad respectiva. Sin embargo, para efecto de la valuación actuarial del fondo no necesita que los resultados se den rápidamente, sino más bien que sean más exactos. 


\begin{tabular}{r|rrrrrrrr}
\hline \hline Año & Activos & $\begin{array}{c}\text { Pens } \\
\text { Total }\end{array}$ & Vejez & Invalidez & Sucesión & $\begin{array}{c}\text { Pens Gen } \\
\text { Inic }\end{array}$ & $\begin{array}{c}\text { Pens Gen } \\
\text { Actual }\end{array}$ & $\begin{array}{c}\text { Pens Gen } \\
\text { Nueva }\end{array}$ \\
\hline 2011 & 65,568 & 493 & 23 & 238 & 232 & 399 & 94 & 0 \\
2015 & 64,512 & 916 & 162 & 552 & 202 & 282 & 634 & 0 \\
2020 & 61,920 & 2,623 & 1,176 & 1,190 & 257 & 207 & 2,416 & 0 \\
2025 & 55,393 & 8,061 & 5,686 & 2,043 & 332 & 155 & 7,906 & 0 \\
2030 & 44,097 & 17,688 & 14,348 & 2,831 & 509 & 112 & 17,576 & 0 \\
2035 & 29,505 & 29,888 & 25,736 & 3,414 & 738 & 86 & 29,802 & 0 \\
2040 & 12,568 & 43,442 & 38,968 & 3,395 & 1,079 & 61 & 43,381 & 0 \\
2045 & 1,308 & 49,949 & 45,665 & 2,896 & 1,388 & 44 & 49,905 & 0 \\
2050 & 23 & 45,254 & 41,175 & 2,321 & 1,758 & 25 & 45,229 & 0 \\
2055 & 0 & 37,992 & 34,227 & 1,726 & 2,039 & 12 & 37,980 & 0 \\
2060 & 0 & 29,776 & 26,541 & 1,191 & 2,044 & 6 & 29,770 & 0 \\
2065 & 0 & 20,997 & 18,576 & 724 & 1,697 & 3 & 20,994 & 0 \\
2070 & 0 & 12,743 & 11,335 & 371 & 1,037 & 0 & 12,743 & 0 \\
2075 & 0 & 6,256 & 5,628 & 149 & 479 & 0 & 6,256 & 0 \\
2080 & 0 & 2,435 & 2,230 & 57 & 148 & 0 & 2,435 & 0 \\
2085 & 0 & 662 & 628 & 10 & 24 & 0 & 662 & 0 \\
2090 & 0 & 106 & 103 & 1 & 2 & 0 & 106 & 0 \\
2095 & 0 & 12 & 12 & 0 & 0 & 0 & 12 & 0 \\
2100 & 0 & 2 & 2 & 0 & 0 & 0 & 2 & 0 \\
\hline \hline
\end{tabular}

Tabla 2: Proyección demográfica salida del SIVA.

\section{Conclusiones}

- El método de cadenas de Markov por Monte Carlo ha sido implementado con éxito para la proyecciones demográficas de las poblaciones que son insumo para las valuaciones actuariales del Régimen de Capitalización Colectiva del sector magisterial de Costa Rica.

- El método de cadenas de Markov por Monte Carlo es recomendable para para la simulación de eventos que siguen distribuciones de Bernoulli, tales como la muerte, invalidez, postergación, entre otros.

- Esta metodología aunque es más lenta que la tradicional (basada en valores esperados), produce más insumos de información para la creación de reportes dentro de la valuación del RCC.

- El método de Monte Carlo ha sido de relevancia para el fondo, pues se generan distintos escenarios posibles de como puede ser una población futura, y esta se simula varias veces con el propósito de obtener un promedio de todos los escenarios y así construir el escenario esperado de cómo se comportará la población en el futuro. 
- El costo/beneficio de la utilización de este método, es positivo ya que a pesar de que para correr sobre una población grande se dure más que en el método tradicional, la generación de información detallada de las proyecciones hace que sea rentable.

\section{Referencias}

[1] Abarca, C. (2000) Historia de la Junta de Pensiones y Jubilaciones del Magisterio Nacional. Editorama, San José, Costa Rica.

[2] Anderson, A.W. (1992) Pension Mathematics for Actuaries. ACTEX Publications, Winsted CT.

[3] Bowers, N.L; Gerber, H.U.; Hickman, J.C.; Jones, D.A.; Nesbitt, C.J. (1997) Actuarial Mathematics. The Society of Actuaries, Chicago IL.

[4] Jordan, C.W. (1967) Life Contingencies. The Society of Actuaries, Chicago IL.

[5] Kellison, S.G. (1991) The Theory of Interest. Irwin/McGraw-Hill, Boston MA.

[6] Ross, S. (1999) Simulation. Academic Press, San Diego CA.

[7] Thullen, P. (1995) Técnicas Actuariales de la Seguridad Social. Ministerio de Trabajo y Seguridad Social, Madrid.

[8] Winklevoss, H. (1993) Pension Mathematics with Numerical Illustrations. Pension Research Council y University of Pennsylvania Press, Philadelphia PA. 
